\title{
INVESTIGACIÓN SOBRE LA APLICACIÓN DE LA ORIENTACIÓN FILOSÓFICA EN LOS CUIDADOS DE SALUD. TERCERA PARTE ${ }^{1}$
}

\author{
Research on the application of Philosophy to Health Care. \\ THIRD PART
}

\author{
FrANCISCO E. BARRERA RODRÍGUEZ \\ Universidad de Sevilla \\ franciscobarrerarodriguez@yahoo.es
}

RECIBIDO: 21 DE OCTUBRE DE 2010

ACEPTADO: 24 DE ENERO DE 2011

Resumen: Este trabajo investiga las vinculaciones entre la Filosofía Aplicada y los cuidados de salud. La primera parte analiza el concepto de salud y prepara la aplicación de la Filosofía al ámbito de la salud. La segunda parte describe los modos en que diversos orientadores filosóficos han llevado a cabo esta labor. La tercera, la aquí expuesta, contiene la propuesta del autor sobre cómo llevar la Filosofía al paciente.

Palabras clave: Filosofía Aplicada, cuidados de salud, orientación filosófica, paciente

\begin{abstract}
This work researches the links between Philosophical Practice and Health Care. First part analyses "Health" concept and prepares to application of Philosophy to Health Care. Second one describes how some philosophical counsellors have undertaken this work. Third part (this one) contains author proposal about how to take philosophy to patient world.
\end{abstract}

Keywords: Philosophical Practice, Health Care, Philosophical Counseling, patient

\footnotetext{
${ }^{1}$ La primera parte de este trabajo fue publicada en el número dos de la Revista Internacional de Filosofía Aplicada HASER en 2011, la segunda en el tercero y esta parte cierra el trabajo completo.
} 


\title{
Una propuesta para la aplicación de la OrFi en cuidados paliativos I: Necesidades espirituales y filosóficas en Cuidados Paliativos.
}

\begin{abstract}
Si la labor del orientador filosófico es la de un "acompañamiento develador"2 dispuesto a quitar la máscara a los impositores desconocidos que determinan nuestra existencia, esta tarea toma aún más sentido cuando se trata de proceder a una relectura de nuestra vida que signifique de la manera más digna y entendida nuestros pasos definitivos hacia el final, nuestro propio reconocimiento en el camino a la muerte. Se propugna, por lo tanto, en la misma intensidad una filosofía para la vida, como un arte de vivir, y una filosofía para la muerte, como un arte de morir. Las culturas del morir han ido cambiando la manera de la muerte a lo largo de la historia ${ }^{3}$. Hemos pasado de una muerte domesticada,
\end{abstract}

\footnotetext{
2 Acompañamiento develador es una tarea propia del orientador filosófico (Implementa la pauta metodológica del Grupo E.T.O.R: oír, escuchar, tratar y derivar) con el individuo o grupo en tanto que de forma acompañada, es decir juntos, sin establecimiento de jerarquías a la manera terapeuta/cliente, se analiza la existencia y sus impositores para hacer de la vida una tarea más consciente, autentica y conocida. También, si es necesario construir desde la crítica reflexionada nuevas propuestas sobre la cosmovisión del individuo a la búsqueda de un sentido que soporte su existencia y la dirija. Este acompañamiento es bidireccional, dado que el orientador viajará en paralelo con el orientado en la misma línea de reflexión y aprendizaje. Esta tarea también se propone de forma especial en el individuo que se encuentra al borde de la muerte y sus familiares. Aquí la relectura de la existencia demanda de una manera más radical un acompañamiento develador a la búsqueda, esta vez, de un sentido para la muerte, en el caso del muriente y de un sentido de aceptación en sus familiares. En ese acompañamiento confluyen los conceptos de la "philia" aristotélica, de la amistad de Shlomit Schuster, además de "asignación al otro", "responsabilidad sin escapatoria" y "paciencia y compasión" de Lévinas.

${ }^{3}$ Cfr. ARIÉS. P.: El hombre ante la muerte. (Original en francés 1977). Taurus, Madrid, 1983. Las actitudes ante la muerte se tornan con el tiempo y son claro espejo de las esperanzas, temores y expectativas del individuo y de la comunidad de su época. El historiador francés nos presenta cuatro actitudes ante la muerte: la doméstica, la de uno mismo, la del otro y la muerte prohibida.

La muerte doméstica, actitud que predominó hasta la Edad Media, era una muerte en la colectividad, sin prisas, con tiempo suficiente para que el muriente releyese su vida y ordenase los asuntos pendientes. Todo se movía de cara a un perdón y a la purificación ante el pecado. El muriente tenía tiempo para despedirse, impartía órdenes y consejos, recibía los últimos sacramentos, hacia testamento y esperaba el final.

La muerte de uno mismo, actitud predominante entre los siglos XII a XV miraba a la parca como individual e íntima. Era una vida sometida a los conceptos judiciales del mundo que se extendían hasta el más allá. Era la soledad del individuo ante la muerte como un pecador, como un sujeto que debería someterse a un dios justiciero y sin piedad. De esta época destacan los pequeños manuales sobre el "Arte de Morir" que instruían al individuo en vida de cómo superar todos los acechos del mal para no sufrir la ira divina.

La muerte del otro, se presenta como una transferencia. Morir se plantea ahora como el último acto de la relación personal con el otro. La transferencia se realizaba normalmente hacia el ser amado. La muerte se mira en el que queda, como su aflicción. Se presenta a la muerte como destino natural, y en torno a ella se construye una simbología y espacios que implican continuamente al abandonado
} 
asumida, esperada, participada por el individuo, su familia y su entorno social, a una muerte prohibida, ajena incluso al muriente porque en su cultura, en su sociedad, la muerte es innombrable; parece no ir con el hombre de la postmodernidad, incapaz de ubicarla en su existencia. La muerte por lo tanto se oculta, se disfraza bajo diferentes mantos para que no enturbie la estética de una existencia que quiere estar ajena al sufrimiento, al dolor y a la condición de finitud que habita en el ser humano.

Esta actitud, tan bien dibujada en las investigaciones de Ariés, nos lleva a enormes problemas a la hora de afrontar la muerte. No sólo porque el sujeto carece del mínimo referente a ella, sino porque tendrá que asumir al extremo su condición de individuo autosuficiente, desprovisto hasta la desnudez del más mínimo equipaje, obvio por otra parte, cuando hablamos del individuo educado desde la postmodernidad.

La medicina del siglo XXI, preocupada en exceso por curar, sabe cómo aliviar las penalidades del proceso del morir, sin embargo, abandona al moribundo, le oculta y esconde de la vida social. Se disimula el duelo, ya no se muere en casa, se han construido tanatorios para guardar bajo edificios perfectamente encajados en la ciudad perfecta, la parte menos deseada de la historia del ser humano, la de su fin. Y lo peor, no se educa para la muerte, ni se habla de ella. Las nuevas generaciones acusan en la actualidad un "no saber" estar ante el sufrimiento, desconocen cómo mostrar a los demás su pesar por la muerte, entre otras cosas, porque esta sociedad se ha encargado de educarlos para que no exterioricen sus sentimientos ${ }^{4}$.

Mostrar los sentimientos es hoy un tabú, así, es muy improbable que los allegados al moribundo le ofrezcan consuelo, apoyo, ternura, estén próximos a él y le toquen, le acaricien, como hacían en la etapa en la que no era un enfermo terminal. Con esta actitud casi le expulsan del mundo de los vivos.

La muerte del individuo hoy es por lo tanto una muerte en soledad, que ha propiciado no sólo la medicina tecnológica, sino las creencias y actitudes sociales asumidas en la postmodernidad. Deshacerse del muriente es pues la primera tarea. Aislarle y separarle del entorno del hogar, y de los seres queridos,

con el partido.

La muerte prohibida es la de nuestro tiempo. En nuestros días la actitud ante ella ha cambiado debido a muchos factores: esperanza de vida mayor por la medicina tecnológica, menor influencia de la religión, desintegración de la familia tradicional. Realmente hay menos conciencia y respeto por la muerte en la actitud contemporánea. En este sentido hay autores que señalan que la preocupación es vivir con calidad la vida, y no vivir para la muerte. La enseñanza de los textos sagrados para la salvación del alma o la buena muerte como el I Ching, el Libro Tibetano de los muertos, el libro egipcio de los muertos y las Ars bene Moriendi han sido sustituidos por la mejor asistencia hospitalaria en la fase terminal.

${ }^{4}$ Cfr.: Elias, N.: La soledad de los moribundos. Fondo de Cultura Económica, México,1987. 
no ir más allá de lo que escuetamente informa el médico que le trata, transferir todo a otros perfectos desconocidos, es la actitud normal en las familias que se enfrentan a la muerte hoy.

Así, confluyen dos vectores que aumentan la línea de fuerza que la normalización del Estado Terapéutico ha dispuesto para estos casos. En el edificio hospitalario manda el médico, y el médico lo sabe. El protocolo es insustituible y las pautas sagradas. El paciente entregado al entramado hospitalario pasa a pertenecer a los rectores de éste, que están aún más legitimados, no ya sólo con la firma protocolaria de los consentimientos informados y con el acceso del enfermo a la carta de sus derechos, meros tics creados para la imagen más liberal y democrática de la política sanitaria de turno, sino que ante el pavor, desconcierto y miedo que produce el enfrentamiento con la muerte, los familiares ponen en manos de la institución sanitaria y por ende del médico a su enfermo, que ya deja de serlo.

En este momento, el muriente es un pecio errante, un ser arrojado a la soledad más terrible, un ser sufriente con muchas necesidades que han de ser satisfechas para no hacer de su último tramo lo peor.

En respuesta a esa situación, donde han confluido otras en las que la obsesión terapéutica ha traspasado los límites de la autonomía del muriente, y donde amparados en determinadas creencias religiosas, se identificaba dolor y sufrimiento como trascendentes y necesarios de aceptar como actitud purificadora para el muriente, surgieron las unidades de Cuidados Paliativos.

Esta medicina paliativa, a la que aún se considera como una medicina de segundo orden, nace de objetivos distintos de la medicina curativa, se desarrolla en torno a la mejora de calidad de vida del muriente. No busca ya la prolongación de la vida a cualquier precio.

Ya no es una lucha contra el tiempo, sino un respeto por éste como campo de enriquecimiento y sentido. En su enfoque integral la medicina paliativa ya no trata al cuerpo como algo fragmentado, propio de la medicina curativa, sino como cuerpo de una persona. Es el dolor físico, el sufrimiento psicológico y el sufrimiento espiritual, lo que Cecily Saunders ${ }^{5}$ denomina "dolor total".

En lo que continúa nos vamos a detener en cómo se satisfacen esas necesidades espirituales, porque nuestra propuesta es que el orientador filosófico forme parte de equipos multidisciplinares de cuidados paliativos para responder a ellas.

\footnotetext{
${ }^{5}$ Fundadora del St.Cristopher's Hospice. Uno de los referentes de los Cuidados Paliativos quien tras la lectura de los trabajos del psiquiatra austriaco Víktor Frankl introdujo en la noción de espiritualidad la búsqueda personal de significado. Cfr. FRANKL.V. El hombre en busca de sentido, op.,cit.
} 
Estas necesidades que hasta el momento se han definido de forma poco clara, acogiendo bajo ese término numerosas preocupaciones que han sido activadas por el enfermo en un momento crucial de su vida en multitud de ocasiones se han querido identificar y reducir a cuestiones simplemente religiosas y en otras sólo a planteamientos existenciales frente a la evidencia de la muerte.

Antes de avanzar en esta dirección se hace imprescindible una reflexión sobre lo que son las necesidades espirituales. En este sentido, Bayés Sopena y Borrás Hernandez ${ }^{6}$ plantean en su interesante trabajo sobre la naturaleza de las necesidades espirituales multitud de interrogantes que habría que despejar para poder afrontarlas de una manera eficaz. Así, estos autores entienden que es necesaria una definición definitiva y consensuada sobre la espiritualidad, disponer de un modelo que permita a los profesionales sanitarios hacer frente a estas necesidades con independencia de sus propios valores y creencias, disponer de herramientas para detectarlas, tener en cuenta la priorización de estas necesidades en cada persona, porque no son idénticas ni universales, estar atentos a la variación en el tiempo de las prioridades de las necesidades que cambian en los enfermos y por último plantean quiénes y cómo deben satisfacer esta necesidad.

No podemos perder de vista que los antecedentes de las unidades de cuidados paliativos están en los hospitales fundados por las órdenes religiosas, de ahí la identificación de estas necesidades con Dios y la religión.

En la declaración fundacional del St. Cristopher's Hospice, que ha servido de modelo a las unidades de cuidados paliativos actuales, puede leerse:

El St. Christopher's Hospice está basado en la fe cristiana en Dios, a través de Cristo. Su objetivo es expresar el amor a Dios a todo el que llega, y de todas las maneras posibles; en la destreza de la enfermería y los cuidados médicos, en el uso de todos los conocimientos científicos para aliviar el sufrimiento y malestar, en la simpatía y entendimiento personal, con respeto a la dignidad de cada persona como hombre que es apreciado por Dios y por los hombres. Sin barreras de raza, color, clase o credo. ${ }^{\text {? }}$

Identificación que como bien recoge el trabajo de Barbero $^{8}$, a propósito del apoyo espiritual en cuidados paliativos, responde a una actitud de comodidad, la

\footnotetext{
${ }^{6}$ BAYÉS, R y BORRÁS F.X.: "Qué son las necesidades espirituales”. Medicina Paliativa, Vol. 12.n 2, Madrid, 2005.

${ }^{7}$ BAYÉS, R.: “¿Debe implicarse el médico en las necesidades espirituales del enfermo en el proceso de morir?" Jano 2005; $\mathrm{n}^{\circ}$ 68, pp. 843-844. (Cursivas del autor).

${ }^{8}$ Cfr. BARBERO, J.: "El apoyo espiritual en cuidados paliativos". Labor Hospitalaria 2002; n 263,
} 
que supone desviar sistemáticamente las cuestiones espirituales a los capellanes o equipos de pastoral.

No es poca la bibliografía que podemos encontrar sobre las necesidades espirituales, ni pocas las investigaciones abiertas en esta línea. Es aquí, precisamente, en la variedad de miradas donde encontramos cierto territorio común que nos sitúa en un espacio de búsqueda personal, de búsqueda de significado de vida. Igualmente no son pocos los autores que hacen uso del término "trascender", es decir, ir más allá, cruzar la frontera, superar al fin, en este caso, la condición de miseria propia del ser humano. Una trascendencia que coloca al individuo en un diálogo con lo sagrado, o consigo mismo en cuanto al proyecto y a la construcción de su vida. El término "sentido", reiteradamente convocado por los estudiosos del tema tiene, como referente al padre de la logoterapia. Autores como Puchalski y Romer ${ }^{9}$ hacen confluir significado y trascendencia, para ellos la espiritualidad permite a la persona una experiencia trascendente del significado de la vida, y si bien puede estar relacionada con lo religioso, también puede relacionarse con la naturaleza, la familia, la comunidad...etc., en la medida en que esos valores dan sentido a su existencia.

La gran mayoría de autores no vincula necesariamente la espiritualidad con lo religioso, sin embargo, sí lo hace con respecto a la búsqueda de sentido, a la confirmación de un significado para la vida examinada, acercándonos de nuevo a propuesta ofrecida por el psiquiatra austriaco Frankl.

Muchas más son las dificultades e interrogantes que nos encontramos en este breve recorrido por las necesidades espirituales en el enfermo terminal: el método para detectarlas, cómo valorarlas, la priorización de éstas con respecto a las necesidades fisiológicas y emocionales...

La profundización en los trabajos de Bayés y Barbero en torno a estas necesidades nos muestran un espacio, ya sugerido, en el que entendemos que el orientador filosófico puede prestar ayuda en el equipo multidisciplinar de cuidados paliativos, siempre que no falte una disposición a ese trabajo en equipo, a un aprendizaje del lenguaje y metodologías de los cuidados paliativos además de una continua implicación en la construcción de proyectos como sugiere uno de estos autores.

Por lo tanto, nos permitiremos exponer lo que entendemos por espiritualidad y cómo desde esta comprensión podemos atisbar esa necesidad filosófica en el paciente, que hasta ahora se ha visto ocultada por el manto del

pp. 5-24.

${ }_{9}^{9}$ Cfr. PUCHALSKI, C. y ROMER, Al.: "Taking a spiritual history allows clinicians to understand patients more fully" Palliat Med, 2000. 3. 
satisfactor espiritual identificado con lo religioso que hacía extensible su función a espacios donde no aportaba nada.

De acuerdo con la OMS en que lo espiritual se refiere a aquellos aspectos de la vida humana que tienen que ver con experiencias que trascienden los fenómenos sensoriales, en que no es lo mismo que lo religioso, y en que va vinculado con el significado y el propósito para los que están cercanos al final de la vida, añadiremos lo expresado en el "Manual de Oxford de Cuidados Paliativos" 10 donde se dibujan tres dimensiones para lo espiritual: trascender lo material, fines y valores últimos y por último el significado existencial.

Reiteramos con otros muchos autores que en esta trascendencia la religiosidad puede ser esencial para estas personas y que en simultáneo, y no necesariamente de forma excluyente pueden convivir con esas demandas ya expresadas y que hasta ahora también han sido incluidas en el apartado de espirituales, si bien los autores a los que esencialmente nos venimos refiriendo en este trabajo, Bayés y Barbero, apuntan espacios que identificamos plenamente como propios de la Filosofía.

Así, Bayés nos propone que junto a las necesidades somáticas, emocionales, sociales y espirituales (a las que circunscribe a la relación del hombre con Dios y continuidad del espíritu después de la muerte), se añadan las existenciales, que englobarían según este autor, las de carácter ético, filosófico y de continuidad biográfica $^{11}$.

Barbero nos propone en sus intervenciones dos posibilidades de derivación. La que ataña a profesionales de "counseling" adecuados y la que corresponde al capellán del "hospice". ${ }^{2}$ Separa en la misma intensidad que Bayés, lo que podemos entender como espiritualidad en la que trascendemos desde lo religioso y espiritualidad que corresponde a una culminación de una existencia que ha convergido en un propósito, en un proyecto, en un sentido. Para esa trascendencia, para esa reflexión, entendemos que la filosofía puede proporcionar interesantes herramientas.

Nuestras divergencias se acentúan con respecto a estos dos psicólogos clínicos en cierta obsesión mostrada en la medición del desfonde espiritual. La necesidad que ambos sugieren de cuantificar el grado de esa necesidad proponiendo, esencialmente en el caso de Barbero, cuestionarios para filtrar y ubicar las citadas necesidades, desgaja un poco ese concepto holístico inherente a la filosofía de los cuidados paliativos, a la vez que tamizan los resultados y los

\footnotetext{
${ }^{10}$ Cfr.: Speck, PW.: "Spiritual issues in palliative care". En: Doyle D, Hanks GWC, Macdonald N. Oxford Textbook of Palliative Medicine. Oxford University Press, Oxford, 1998.

${ }^{11}$ Ibid., p. 102.

${ }^{12}$ Ibid.
} 
reconducen, perdiéndose en este camino una parte muy importante de la intensidad que buscamos conocer.

Detectar estas necesidades, valorarlas, priorizarlas sobre las demás y decidir quién las atiende finalmente, es un gran problema, estamos de acuerdo, como también lo estamos en que no es trabajo de una sola mirada, sino de todas las perspectivas que puedan aportar satisfactores al muriente y a sus allegados.

En este punto se propone una intervención en el grupo interdisciplinar del orientador filosófico, en tres esferas que están directamente relacionadas y que participan en simultáneo en la puesta en escena del final de una vida.

\begin{tabular}{|c|c|}
\hline Con el enfermo & $\begin{array}{l}\text { - } \text { Relectura vital. Biografía. Mis } \\
\text { significantes existenciales. } \\
\text { - } \quad \text { Análisis de los valores. } \\
\text { - Construcción de un sentido de } \\
\text { vida hacia la muerte. }\end{array}$ \\
\hline Con su familia & $\begin{array}{l}\text { - } \text { Relectura vida en común. } \\
\text { Biografía grupal. } \\
\text { - } \text { Ante la pérdida. Cómo afrontarla } \\
\text { con sentido. } \\
\text { - Construcción del duelo. }\end{array}$ \\
\hline Con el personal Sanitario & $\begin{array}{l}\text { - Oír y escuchar. Del paciente a la } \\
\text { persona. Identificar las cuestiones } \\
\text { existenciales. } \\
\text { - Análisis de emociones ante el } \\
\text { paciente. Reconstruir las pautas. } \\
\text { Yo. El equipo (Diversidad de } \\
\text { miradas). }\end{array}$ \\
\hline
\end{tabular}

El orientador filosófico, de acuerdo con lo expresado por Bayés cuando dice:

En cuanto a la metodología, cada vez estoy más convencido de que para el estudio de la evolución de la realidad subjetiva de los enfermos que se acercan al final de su existencia, los cuestionarios y las escalas de respuesta cerrada cortan la comunicación y, probablemente, impiden el acceso al conocimiento de las verdaderas preocupaciones del enfermo al que se desea ayudar. ${ }^{13}$

\footnotetext{
${ }^{13}$ Ibid.
} 
propugna un "proceso dialógico", un oír para luego escuchar desde un espacio de acompañamiento, llevando al extremo la acepción de acompañar que dice: "existir junto a otra o simultáneamente con ella"", no cosa, en este caso, sino persona que se prepara para la muerte. Desde ese "acompañamiento develador" antes propuesto, el recorrido del orientador debe empaparse en la "filia" aristotélica, de la amistad en el recorrido propuesta por el filósofo israelí Lahav, desde las metáforas de Enmanuel Lévinas ante la vulnerabilidad radical del otro. Nada nuevo bajo el sol, pero por evidente olvidado. Nada más necesario que recibir del otro la compañía. La soledad de la muerte, nos recuerda el filósofo lituano, no hace desaparecer al prójimo, nos insiste que hay lugar todavía para una llamada al otro, a su amistad. ${ }^{15}$

La labor del filósofo no debería circunscribirse en el campo de la medicina a la de ocupar sillones en comités éticos donde se presente a éste como una autoridad en conocimiento moral. Comparto con Victoria Camps ${ }^{16}$ la imposibilidad de sostener este estatus.

Lejos de reforzar la estructura burocrática del Estado Terapéutico que nos presenta hoy a los comités éticos como un avance democrático y un ajuste necesario hacia la excelencia de nuestra sanidad, habría que reflexionar a fondo hasta qué punto puede implicarse alguien que no baje al terreno de la práctica, donde habita la carne, la decisión urgente, la fragilidad del pálpito de quien bordea el final.

El filósofo no está para asentir en el consenso, ni para asesorar al individuo a familiares o a personal sanitario, sino para provocar la reflexión conjunta de todos los participantes en este duro proceso.

El acompañamiento, acoge la humildad y la compasión (la del ser humano por el ser humano), pero debe acoger igualmente la provocación ante toda normalización. Como sugiere Vaughana Feary ${ }^{17}$ : ayudar a un mejor afrontamiento, mejora de toma de decisiones y asumir responsabilidades, concienciar a los enfermos de la necesidad de demandar y crear comunidades terapéuticas y sistemas de salud más justos. Apoyar la autonomía del paciente, incluyendo el derecho a morir

\footnotetext{
14 Cfr. http://buscon.rae.es/draeI/SrvltGUIBusUsual?TIPO_BUS=3\&LEMA=acompañar (Último acceso 5 septiembre 2006).

${ }^{15}$ Cfr. Lévinas, E.: Totalidad e Infinito. Ensayo sobre la exterioridad. (Traducción de Daniel.E. Guillot). Sígueme, Salamanca, 2002.

${ }^{16}$ Cfr. Camps, V.: Una vida de calidad. Reflexiones sobre bioética. Ares y Mares, Barcelona, 2001.

${ }^{17}$ Ibid., p. 2.
} 


\section{Una propuesta para la aplicación de la OrFi en cuidados paliativos II: trabajo de campo sobre "necesidades filosóficas".}

FORMULARIO DE ENCUESTA A MEDICOS DE CUIDADOS PALIATIVOS PARA VALORAR "NECESIDADES FILOSÓFICAS DE LOS PACIENTES"

\begin{tabular}{|l|l|}
\hline Médicos Cuidados Paliativos -3 años servicio & \\
\hline Médicos Cuidados Paliativos +3 años servicio & \\
\hline
\end{tabular}

1) Si tuviera que definir esta necesidad ¿cómo lo haría?

2) ¿La diferencia de otras necesidades del enfermo que puedan parecer similares?
a Psicológicas
b Emocionales
c Espirituales
d Religiosas

\begin{tabular}{|l|l|l|}
\hline $\mathrm{Si}$ & No & No sabe \\
\hline & & \\
\hline & & \\
\hline & & \\
\hline & & \\
\hline
\end{tabular}

3) ¿ Detecta en su trabajo diario esta necesidad?

\begin{tabular}{|l|l|l|}
\hline Si & No & No sabe \\
\hline & & \\
\hline
\end{tabular}

4) ¿Desarrolla algún proceso específico de evaluación para detectar estas necesidades entre sus pacientes?
a Diálogo
b Cuestionarios
c Observación
d Información de familiares
e Esperar que las manifieste el paciente espontáneamente

\begin{tabular}{|l|l|l|}
\hline Frecuentemente & Nunca & $\begin{array}{l}\text { A } \\
\text { veces }\end{array}$ \\
\hline & & \\
\hline & & \\
\hline & & \\
\hline & & \\
\hline & & \\
\hline
\end{tabular}


5) ¿Cómo se afrontan estas necesidades habitualmente?

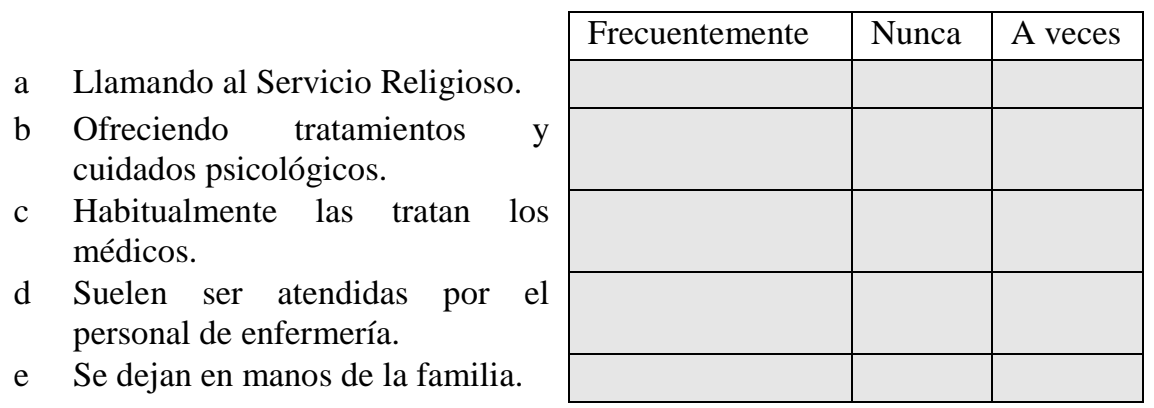

6) Conoce la figura del "philosophical counselor" y/o del "orientador filosófico"?

\begin{tabular}{|l|l|l|}
\hline $\mathrm{Si}$ & No & No sabe \\
\hline & & \\
\hline
\end{tabular}

7) ¿Consideraría adecuada la figura de este profesional para la atención de las necesidades filosóficas de los pacientes en situación terminal formando parte de un equipo multidisciplinar?

\begin{tabular}{|l|l|l|}
\hline Si & No & No sabe \\
\hline & & \\
\hline
\end{tabular}

En caso afirmativo en el anterior cuestionario responder al siguiente:

Si es la figura adecuada

No es la figura adecuada

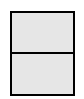

Trabajo de campo. ¿Qué nos ayuda a morir mejor? Algunas consideraciones.

El presente trabajo se desarrolló sobre una muestra de 200 encuestas realizada entre los meses de enero y diciembre de 2006 en la ciudad de Sevilla.

Se tomó como base para su realización un interesante trabajo realizado por un equipo ${ }^{18}$ formado por miembros de la Facultad de Psicología de la UAB y médicos del Servicio de Hematología y Hemoterapia del Hospital La Paz de

\footnotetext{
${ }^{18}$ ARRANZ, P., BAYÉS, R., LIMONERO, J. y ROMERO, Eva.: “¿Qué puede ayudarnos a morir en paz?" Medicina Clínica. 2000, (15), pp. 579-582.
} 
Madrid, que a su vez había tomado como base los resultados del trabajo de Singer et al. ${ }^{19}$

El trabajo del equipo español desarrolló la encuesta en el ámbito sanitario, circunstancias que hemos eludido en este trabajo con la intención de eliminar las posibles inferencias en las respuestas de consultados que por su profesión vivían directamente, y de forma consciente, con el morir.

El trabajo de campo se presenta fundamentalmente como inicio de una futura investigación sobre el tema. Para el trabajo que nos ocupa se han extraído cuatro ítems que valoran fundamentalmente:

- Valoración sobre el sentido de la vida ante la inmediatez de la muerte.

- Actitud ante el sufrimiento.

- Valoración de la autonomía personal a la hora de tomar decisiones ante la propia muerte.

\section{MUESTRA MAYORES DE 65 AÑOS}

\begin{tabular}{|l|r|r|}
\hline si & $77,70 \%$ & 7 \\
\hline no & $22,30 \%$ & 2 \\
\hline
\end{tabular}

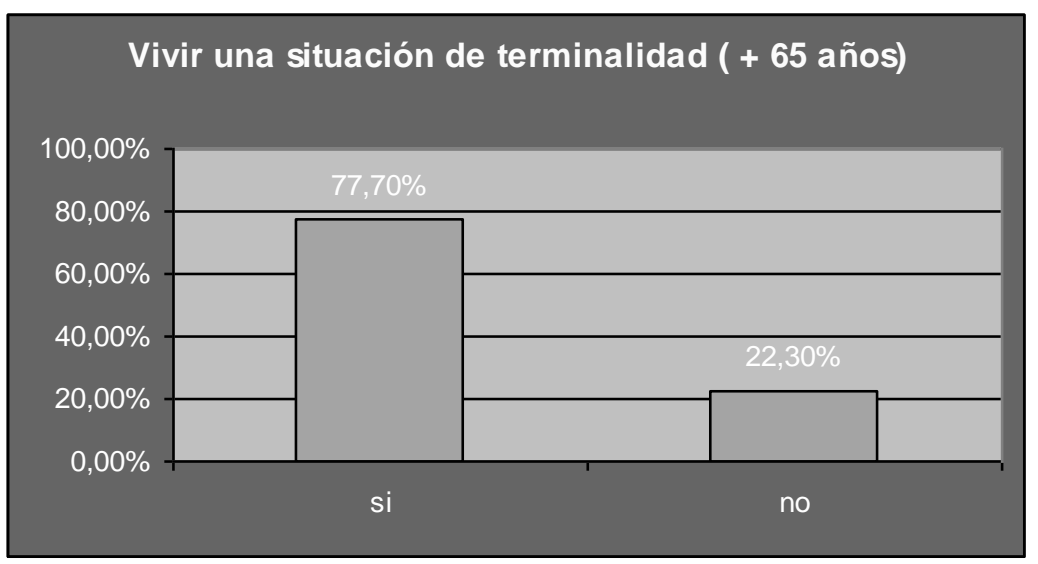

19 SINGER, P. A., MARTIN, D, K. y KELNER, M.: “Quality end of-life care”. JAMA 1999, (281), pp. 163-168. 


\begin{tabular}{|l|r|r|r|}
\hline \multicolumn{1}{|c|}{ Items seleccionados } & \multicolumn{1}{|c|}{ Nada } & \multicolumn{1}{c|}{ Algo } & \multicolumn{1}{c|}{ Mucho } \\
\hline Autonomía para decidir & 2 & & 7 \\
\hline No sufrir más de lo necesario & & & 9 \\
\hline $\begin{array}{l}\text { Pensar que la vida tuvo un } \\
\text { sentido }\end{array}$ & & 2 & 7 \\
\hline Una muerte rápida. & & 1 & 8 \\
\hline \multicolumn{1}{|c|}{ Items seleccionados } & Nada & Algo & \multicolumn{1}{c|}{ Mucho } \\
\hline Autonomía para decidir & $22,30 \%$ & & $77,70 \%$ \\
\hline No sufrir más de lo necesario & & & $100 \%$ \\
\hline $\begin{array}{l}\text { Pensar que la vida tuvo un } \\
\text { sentido }\end{array}$ & & $22,20 \%$ & $77,80 \%$ \\
\hline Una muerte rápida. & & $11.20 \%$ & $88,80 \%$ \\
\hline
\end{tabular}

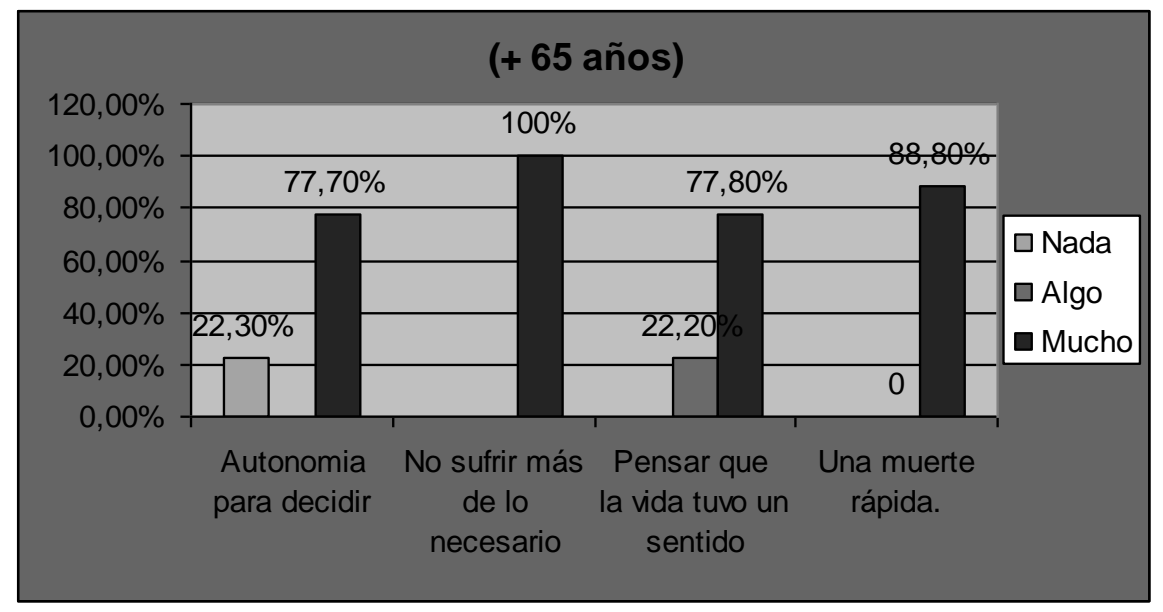


MUESTRA ENTRE 40 Y 65 AÑOS

\begin{tabular}{|l|r|r|}
\hline si & $69,80 \%$ & 60 \\
\hline no & $30,20 \%$ & 26 \\
\hline
\end{tabular}

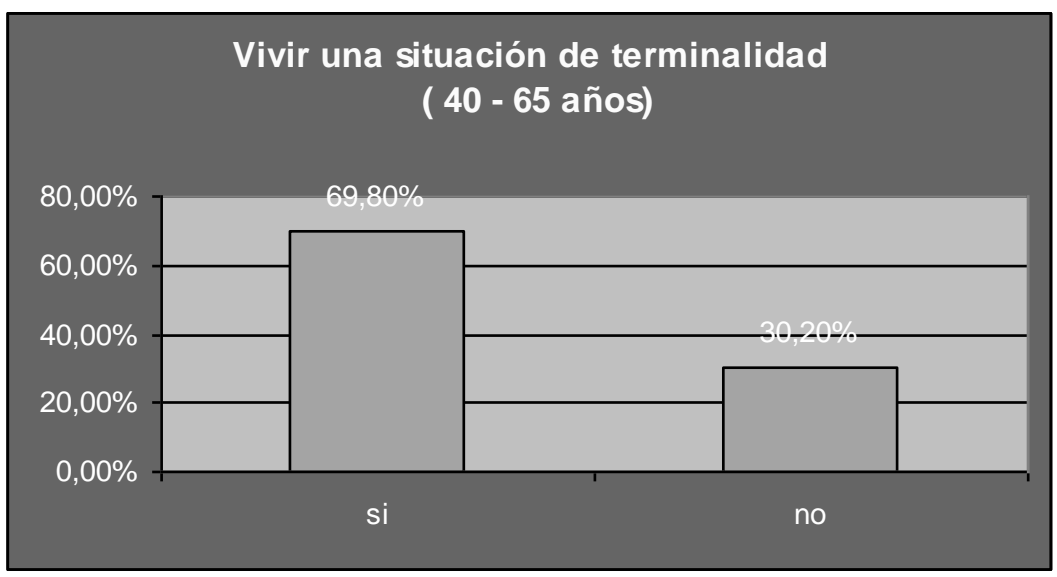

\begin{tabular}{|l|r|r|r|}
\hline \multicolumn{1}{|c|}{ Items seleccionados } & \multicolumn{1}{c|}{ Nada } & \multicolumn{1}{c|}{ Algo } & \multicolumn{1}{c|}{ Mucho } \\
\hline Autonomía para decidir & 12 & 30 & 44 \\
\hline No sufrir más de lo necesario & 1 & 4 & 81 \\
\hline $\begin{array}{l}\text { Pensar que la vida tuvo un } \\
\text { sentido }\end{array}$ & 9 & 10 & 67 \\
\hline Una muerte rápida. & 10 & 13 & 63 \\
\hline \multicolumn{1}{|c|}{ Items seleccionados } & Nada & \multicolumn{1}{c|}{ Algo } & Mucho \\
\hline Autonomía para decidir & $13,93 \%$ & $34,90 \%$ & $51,17 \%$ \\
\hline No sufrir más de lo necesario & $1,20 \%$ & $4,70 \%$ & $94 \%$ \\
\hline $\begin{array}{l}\text { Pensar que la vida tuvo un } \\
\text { sentido }\end{array}$ & $10,50 \%$ & $11,70 \%$ & $77,80 \%$ \\
\hline Una muerte rápida. & $11,70 \%$ & $15,10 \%$ & $73,20 \%$ \\
\hline
\end{tabular}




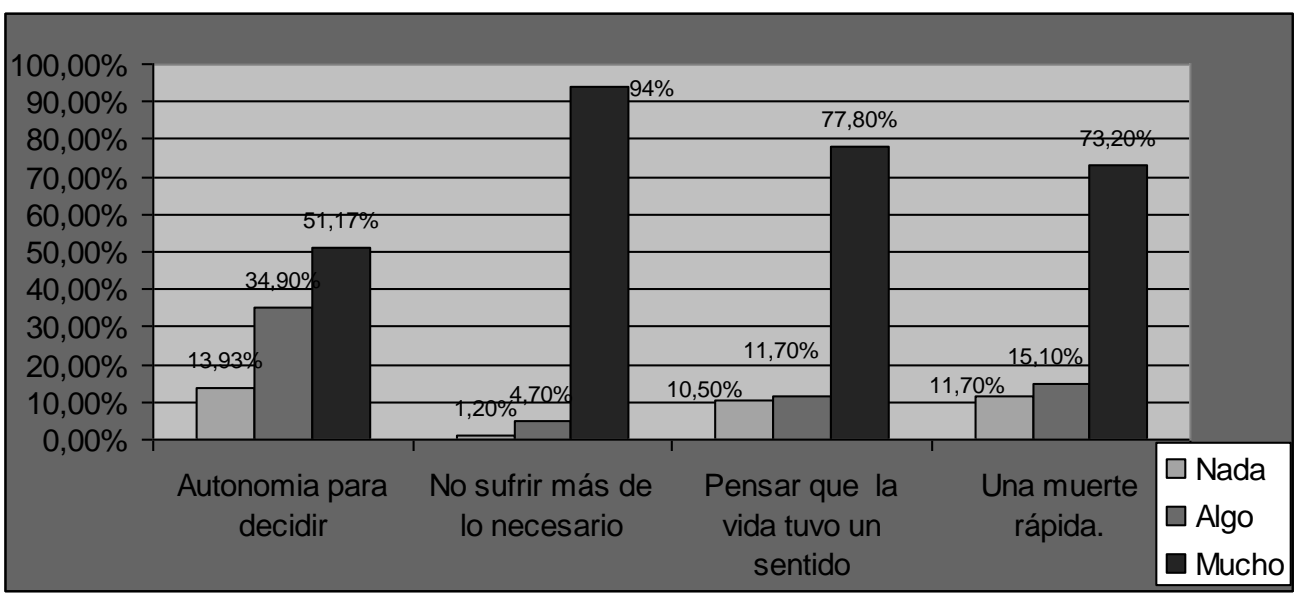

MUESTRA MENOS DE 40 AÑOS

\begin{tabular}{|l|r|r|}
\hline si & $53,40 \%$ & 56 \\
\hline no & $46,60 \%$ & 49 \\
\hline
\end{tabular}

\section{Vivir una situación de terminalidad (- de 40 años)}

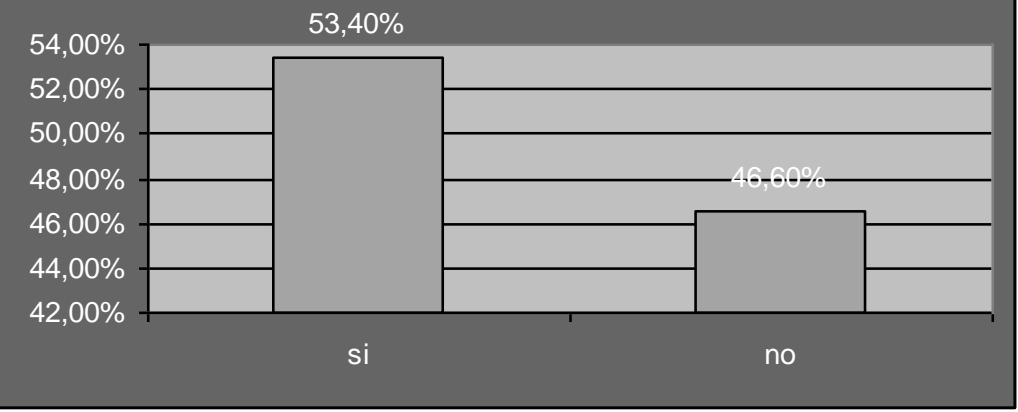




\begin{tabular}{|l|r|r|r|}
\hline \multicolumn{1}{|c|}{ Items seleccionados } & \multicolumn{1}{c|}{ Nada } & \multicolumn{1}{c|}{ Algo } & \multicolumn{1}{c|}{ Mucho } \\
\hline Autonomia para decidir & 15 & 30 & 60 \\
\hline No sufrir más de lo necesario & 4 & 7 & 94 \\
\hline Pensar que la vida tuvo un sentido & 7 & 25 & $\mathbf{7 3}$ \\
\hline Una muerte rápida. & 8 & 12 & $\mathbf{8 5}$ \\
\hline \multicolumn{1}{|c|}{ Items seleccionados } & Nada & \multicolumn{1}{c|}{ Algo } & \multicolumn{1}{c|}{ Mucho } \\
\hline Autonomia para decidir & $14,30 \%$ & $28,60 \%$ & $57,10 \%$ \\
\hline No sufrir más de lo necesario & $3,80 \%$ & $6,60 \%$ & $90 \%$ \\
\hline Pensar que la vida tuvo un sentido & $6,60 \%$ & $23,80 \%$ & $69,60 \%$ \\
\hline Una muerte rápida. & $7,60 \%$ & $11,40 \%$ & $81,00 \%$ \\
\hline
\end{tabular}

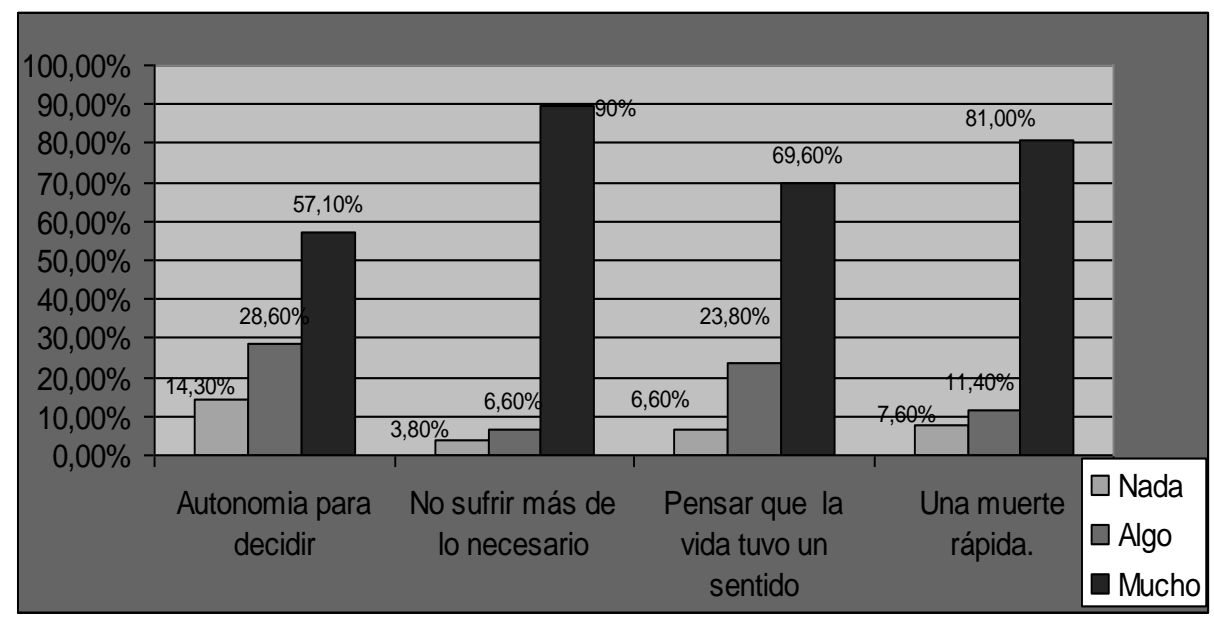


TOTAL MUESTRA

\begin{tabular}{|l|r|r|}
\hline si & $61.50 \%$ & 123 \\
\hline no & $46,60 \%$ & 77 \\
\hline
\end{tabular}

\section{Vivir una situación de terminalidad MUESTRA TOTAL}

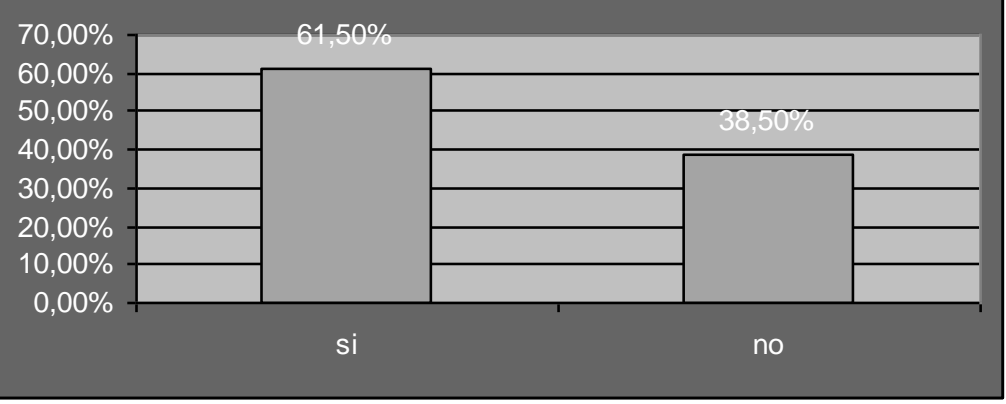

\begin{tabular}{|l|r|r|r|}
\hline \multicolumn{1}{|c|}{ Items seleccionados } & \multicolumn{1}{c|}{ Nada } & \multicolumn{1}{c|}{ Algo } & \multicolumn{1}{c|}{ Mucho } \\
\hline Autonomia para decidir & 29 & 60 & 111 \\
\hline No sufrir más de lo necesario & 5 & 11 & 184 \\
\hline $\begin{array}{l}\text { Pensar que la vida tuvo un } \\
\text { sentido }\end{array}$ & 16 & 37 & 147 \\
\hline Una muerte rápida. & 18 & 26 & 156 \\
\hline \multicolumn{1}{|c|}{ Items seleccionados } & Nada & \multicolumn{1}{c|}{ Algo } & Mucho \\
\hline Autonomia para decidir & $14,50 \%$ & $30,00 \%$ & $55,50 \%$ \\
\hline No sufrir más de lo necesario & $2,50 \%$ & $5,50 \%$ & $92 \%$ \\
\hline $\begin{array}{l}\text { Pensar que la vida tuvo un } \\
\text { sentido }\end{array}$ & $8,00 \%$ & $18,50 \%$ & $73,50 \%$ \\
\hline Una muerte rápida. & $9,00 \%$ & $13,00 \%$ & $78,00 \%$ \\
\hline
\end{tabular}




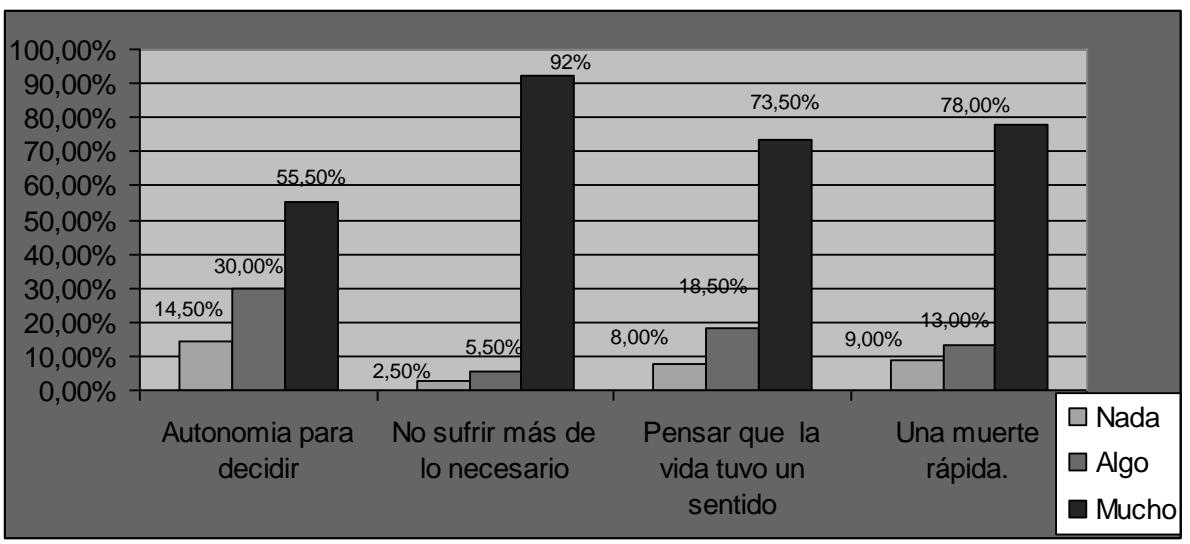

\section{Conclusiones}

Como reseñábamos en la introducción de este trabajo, no era nuestra intención reiterar en los beneficios de la OrFi, entendiendo a ésta como la aplicación de la filosofía a los avatares del ser humano en su trayectoria existencial para procurar una vida satisfactoria que traduciríamos en: vivir una vida más pensada, más controlada y más autónoma.

Concluir no debería ser repetir, y no quiero reincidir en lo ya expuesto, si bien, sí reseñar muy brevemente y casi de manera telegráfica, algunas ideas que creo pueden fijar definitivamente la propuesta que se hace en este trabajo de investigación, que no va más allá de mostrar, que la filosofía en su desarrollo como Orfi tiene una aplicación concreta en el campo de la salud.

Este trabajo en la salud pasa necesariamente por un método de trabajo que se sustenta en los siguientes elementos:

- Los propios del método ETOR: Oír, Escuchar, Tratar y Derivar.

- Los que implementa la Logoterapia: Derreflexión, Intención Paradójica y Diálogo Socrático.

- La actitud definida como de "acompañamiento develador". Donde se incluye la concepción de amistad griega que nos conduciría a un compartir la virtud (la de una vida releída y pensada en cualquiera de sus aspectos, y esencialmente en los del sufrimiento y el afronte del morir).

Desde estas pautas metodológicas, proponemos que el orientador filosófico atienda un doble papel en el desarrollo de la OrFi de cara a la salud. El de 
provocar y promover la reflexión de todos los individuos de la sociedad sobre la condición finita del ser humano y en consecuencia su condición de individuo arrojado al dolor y sufrimiento que debe afrontar como parte real y consustancial del existir. Reclamar junto a éstos la necesidad de una sanidad justa y equitativa que se ajuste fundamentalmente a los deseos del paciente, que éste sea tratado como persona y no como caso, además de demandar junto a estos unos fines de la medicina que no antepongan el curar al cuidar. El filósofo debe participar activamente en las denuncias que alertan sobre la medicalización de la salud y el negocio de las industrias farmacéuticas con el dolor y el sufrimiento de los pobres, (patentes, vacunas, genéricos...etc.).

En el dolor y el sufrimiento ayudar a un mejor afrontamiento, toma de decisiones y a asumir responsabilidades. Acompañar en la reflexión sobre las necesidades filosóficas que se planteen en enfermos cercanos al morir y que difieren de las religiosas, si bien éstas no son excluyentes. Apoyar la autonomía del paciente y su derecho a decidir sobre la muerte.

Finalizar subrayando que el filósofo de nuestra sociedad no debe cerrarse exclusivamente a la relectura y reinterpretación del corpus filosófico oficialmente aceptado. Debe abrirse a otras propuestas y sobre todo propiciar la reflexión en unos días en los que la sociedad de consumo la persigue sin contemplaciones en su afán de multiplicar adictos del estado-ganadero.

La normalización, hoy justificada y democratizada, debe recibir propuestas críticas y constructivas para que los individuos no mueran del éxito total de la sociedad del bienestar que abre enormes fracturas y desigualdades en una estrategia perfectamente urdida por aquellos que viven del sufrimiento de los otros.

Si ésta es la llamada a los filósofos académicos, debe quedar para los que arremeten con sus nuevas propuestas, que esta llamada a la filosofía a campar por la plaza pública no quede en más que una nueva terapia para malestares de burgueses con ciertas preocupaciones pseudofilosóficas que finalicen en crisis de sentido, y terminen convirtiéndose en otros terapeutas para los malestares de la postmodernidad. 
